\title{
Separation thresholds for colored bars with varied luminance contrast'
}

HAROLD P, BISHOP

TUFTS UNIVERSITY

Separation threshold scores were obtained for colored rectangular bar targets of constant luminance presented against a white ground varied in luminance. Maximum separation scores were obtained at nominal zero luminance contrast with red and blue targets; and at greater than nominal zero luminance contrast with yellow and green targets.

The marked deterioration of visual acuity with loss of luminance contrast for black and white stimulus configuration is well known. Other work (e.g., Cavonius \& Schumacher, 1965), as well as data previously reported by the author (1966) suggests, however, that quite good acuity scores may be obtained with stimulus configurations which present color contrast without luminance contrast. With colored bars equated in luminance to the white ground, minimum separation scores on the order of $0.6 \mathrm{~min}$. of visual angle were obtained. The lowest scores, obtained with black targets on colored grounds and nominal $100 \%$ luminance contrast, were of the order of $0.5 \mathrm{~min}$. The data suggested the hypotheses that separation scores are maximum with color contrast only and would show a regular decrease with increase in luminance contrast. A practical consideration suggested here is that maximum separation, minimum acuity, or some variation might be used as the critical discrimination in heterochromatic photometry. Initial data bearing on these questions is presented below.

\section{Subjects}

One female and two males, ages 21 to 37 , served in the experiment. The two males wore corrective lenses and all Ss scored 1.0 or better in the far and near visual acuity tests on the AO Orthorater. All Ss scored $100 \%$ on the Ishihara and AO tests for color deficiency. Method

The test stimulus pattern was a pair of rectangular bars which subtended approximately $0.5^{\circ} \times 1.1^{\circ}$ of visual angle at $63 \mathrm{~cm}$ presented against a $7^{\circ}$ ground. A larger annular surround increased the illuminated field to $22^{\circ}$. The target, ground and surround were independently illuminated from small diffuse sources with the light directed to the eye in a modified Maxwellian manner. The targets, ground and surround were equated at 5 ft. - L. The calibration procedure was as follows: A Weston photronic cell equipped with a viscor filter was calibrated against flicker matches of each color against white for each $\mathrm{S}$. The ground luminance was set for each color with use of the photronic cell, and the target levels determined by homochromatic matches of the targets against the grounds. The color filters used, with their calculated dominant wavelengths were: C-(Corning) 2-59, $640 \mathrm{~nm}$; Wratten No. 73, $571 \mathrm{~nm}$; C-4-64, $528 \mathrm{~nm}$; $\mathrm{C}-5-60,458 \mathrm{~nm}$.
Before an experimental session the Swas dark adapted for $1 / 2 \mathrm{hr}$. He was then positioned at the apparatus and light adapted for $5 \mathrm{~min}$. to white light at $5 \mathrm{ft}$. $-\mathrm{L}$. After adaptation the $S$ was given 10 practice adjustments with white bars presented on a black ground. He was then adapted to white for $2 \mathrm{~min}$. after which the first test condition was presented. Observation was monocular through a $3 \mathrm{~mm}$ artificial pupil with head and eye position controlled by means of an adjustable bite board bearing the S's dental impression.

The task for the $S$ was to make two sorts of adjustments of the target bar pair. Starting with the bars well apart he brought them together to the point at which the line of separation just was not seen, a together judgment. This setting was read from a calibrated dial on the target carriage mechanism and the targets were then moved more closely together. The $S$ then moved the targets apart until he just perceived a line of separation, an apart judgment. Ss were allowed to bracket the judgment point but were restricted to making the last movement in the indicated direction. The mean of the pair of judgments was taken as the threshold score. The Ss made five pairs of judgments for each stimulus combination with a $1-\min$ a adaptation to white light between each combination. Threshold scores were obtained for all combinations of target and ground luminance for a given color during a single session; and four counterbalanced replications were obtained for each color with each S.

\section{Results}

The data obtained with one $S$ is shown in Fig. 1. Data for other Ss was similar with differences in detail. The curves show the threshold scores obtained with red, yellow, green and blue targets seen against a white ground at various luminance contrast levels. Luminance contrast was arbitrarily designated to be positive for conditions including a ground luminance below that of the targets and negative when it was above the target level. The curves are arbitrarily separated vertically. Each data point is the mean of 20 pairs of judgments and variability of judgments is shown by the vertical line through the data points indicating the limits of one standard deviation on either side of the mean. With all target colors separation scores are seen to rise to a maximum and then to decrease as the luminance of the ground was varied through values below to values above that of the target. With the blue and red targets maximum separation scores were obtained with zero luminance contrast. With yellow and green targets maximum separation scores were obtained with the background luminance above that of the target. 


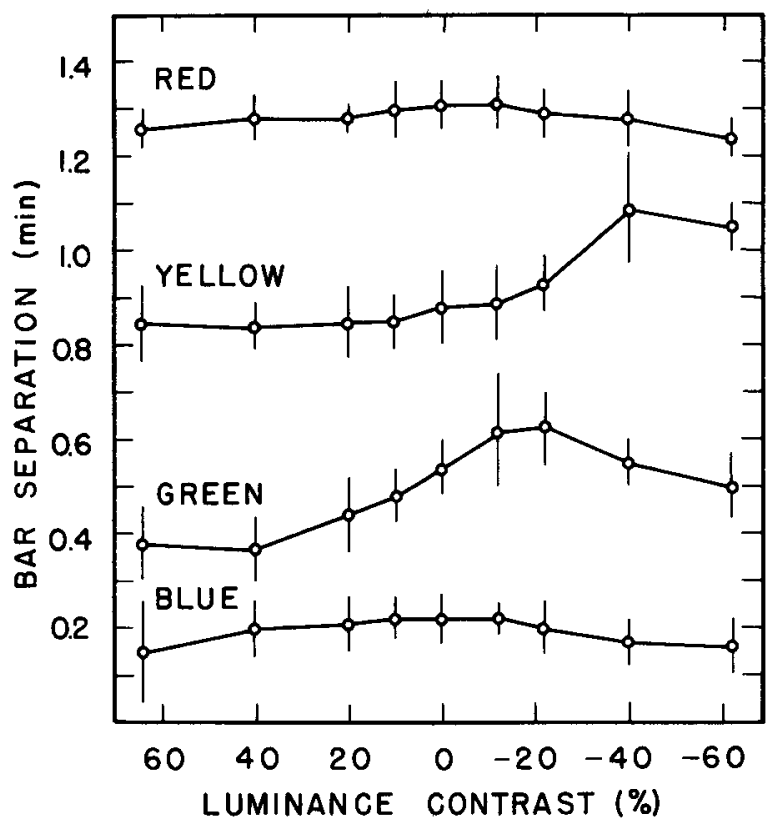

Fig. 1. Mean detectable bar separation of $0.5^{\circ} \times 1.1^{\circ}$ colored bars presented on a white ground varied in luminance. Curves are arbitratily separated. Variability of the scores is shown by vertical lines indicating the limits of one standard deviation on either side of the mean score.

\section{Discussion}

The results obtained with the red and blue targets are in accord with the prediction of maximum separation scores at zero luminance contrast. The slow change in the scores with change in luminance contrast with the present targets, however, indicates this type of discrim- ination to be of little use as a photometric technique.

The failure to obtain a similar maximum separation score at zero luminance contrast with the yellow and green stimuli is probably related to the chromatic aberration and the state of accommodation of the eye when making judgments with these stimuli. A check of the luminance calibrations revealed no apparent error. A check set of data obtained with yellow targets and ground equated in a direct homochromatic match showed the same pattern. A crude check of the effects of the state of accommodation was accomplished with use of a minus lens of low dioptric power interposed between the eye and the stimuli. Small changes in accommodation were found to have a more or less marked effect on the appearance of the configuration at threshold, and upon the obtained scores, depending on the a mount and direction of the nominal target-ground luminance contrast. Cavonius and Schumacher, who used a grid target, also noted that acuity scores were depressed with the addition of luminance contrast with certain target-ground color combinations. Further exploration of this problem is under way.

\section{References}

Bishop, H. P. Separation thresholds for colored bars with and without luminance contrast. Psychon. Sci., 1966, 4, 223-224.

Cavonius, C. R., \& Schumacher, A. W. Human chromatic visual acuity. J. Opt. Soc. Amer., 1965, 55, 1589.

\section{Note}

1. The research reported here was supported by United States Public Health Service Research Grant NB 05088-02 from the National Institutes of Health, Division of Neurological Diseases and Blindness. 